\title{
THE ANALYSIS OF DIFFUSION CONTROLLED REACTIONS \\ WITH NON-EQUAL DIFFUSIVITIES OF THE REACTANTS
}

\author{
Amable LIÑAN \\ Escuela Técnica Superior de Ingenieros Aeronáuticos \\ Universidad Politécnica de Madrid
}

\begin{abstract}
We show, using as a model problem the description of the reaction of a fuel pocket with the oxygen of the environment, how to derive a system of conservation equations to describe the evolution of the temperature and concentrations of the reactants, when these are not initially mixed, or only partially mixed, and the characteristic reaction time is very short compared with the characteristic diffusion time.
\end{abstract}

\section{Introduction and formulation}

In the analysis of reactive systems, in particular of combustion systems, we find that the characteristic reaction time, $t_{c}$, is often very short when compared with the diffusion time, $t_{d}$, or with the residence time in the chamber. Then, the reactants (the fuel and oxygen in combustion systems), if fed independently into the reaction chamber, can only coexist, and with small concentrations, in thin layers (the diffusion flames of combustion systems), where the chemical reaction takes place when the reactants arrive by diffusion from opposite sides of the layer. The products and heat liberated in the layer by the reaction diffuse out in opposite direction to the reactants; their peak concentrations and the peak value of the temperature, reached at the thin layer, is determined by the relative values of the thermal and mass diffusivities of the reactants and products.

The reaction layer becomes infinitely thin in what we call the Burke-Schumann 
limit of infinite chemical reaction rate; namely, in the limit of infinite Damköhler numbers, $t_{d} / t_{c} \rightarrow \infty$. The position of the reaction surface is determined by the requirement that the reactants must reach the surface in stoichiometric proportions and have zero concentration there. The problem of determining the position of the flame surface is a free-boundary problem, which becomes extremely difficult when, as desirable in combustion systems, the flow is turbulent; then, the velocity fluctuations give a strongly wrinkled fractal character to the flame surface.

Burke and Schumann (1928) showed how the problem of determining the flame surface, and the temperature and concentration of the reactants, could be simplified when the diffusivities of the reactants, $D_{F}$ and $D_{0}$, are equal to the thermal diffusivity, $D_{T}$. In this case, we find linear combination of the reactant concentrations and temperature, also called Schvab-Zeldovich coupling functions, not changed by the reactions, which diffuse through the chamber as conserved scalars. One normalized form of these conserved scalar is the mixture fraction $Z$, widely used in the analysis of turbulent combustion systems, defined so as to be zero in the air feed stream and 1 in the fuel feed stream. See Williams (1985).

The purpose of this note is to show how it is possible to generalize the BurkeSchumann technique to deal with cases where the Lewis numbers, $L_{F}=D_{T} / D_{F}$ for the fuel and $L_{0}=D_{T} / D_{0}$ for the oxygen, are different from unity. For earlier presentatios of this generalization see Liñán (1991a) and (1991b) and Liñán and Williams (1993).

In this note, for simplicity in the presentation, we shall leave out the effects of convection, and thus limit ourselves to the analysis of reactive-diffusive systems. We shall illustrate the method by analysing the evolution with time of a pocket of fuel, of size $L$, in an unbounded air environment.

If the characteristic reaction time $t_{c}$ is very small compared with the characteristic diffusion time $t_{d}=L^{2} / D_{T}$, we shall find two stages in the evolution of the system. A first stage, for times $t \sim t_{c}$, where if the reactants are initially mixed the reaction will take place, without effects of diffusion, until complete depletion of either the fuel or the oxygen. Then, in a second much longer stage, the fuel 
remaining in the core of the fuel pocket will diffuse out through the products to meet the oxygen, coming in the opposite direction. The reaction will occur in a thin reaction layer that will shrink to diseppear in a time of order $t_{d}$.

We shall consider an irreversible chemical reaction of the type

$$
\mathrm{F}+s \mathrm{O}_{2} \rightarrow(1+s) P_{r}+(q)
$$

where $s$ mass units of oxygen are consumed per unit mass of fuel to generate $(1+s)$ mass units of products and a quantity $q$ of heat. Although it is not essential for the results of the analysis of the second stage, we shall consider that the mass consumption rate of fuel per unit volume and time, $W_{F}$, follows the Arrhenius law

$$
W_{F}=\rho B e^{-E / R T} Y_{F 0} Y_{0 \infty} y_{F} y_{0}
$$

where $y_{F}$ and $y_{0}$ are the mass fractions of the fuel and oxygen, measured with their maximum initial values $Y_{F 0}$ and $Y_{0 \infty} . B$ is a frequency factor, $E$ is the activation energy, $R$ is the gas constant, $\rho$ the density, and $T$ the temperature.

The conservation equations for the mass fractions $y_{F}$ and $y_{0}$ and the temperature, if the density, $\rho$, specific heat, $c_{\boldsymbol{p}}$, and diffusivities are assumed to be constant, take the form

$$
\begin{gathered}
\frac{\partial}{\partial t} y_{F}-\frac{D_{T}}{L_{F}} \Delta y_{F}=-\frac{W_{F}}{\rho Y_{F 0}} \\
\frac{\partial}{\partial t} y_{0}-\frac{D_{T}}{L_{0}} \Delta y_{0}=-S \frac{W_{F}}{\rho Y_{F 0}} \\
\frac{\partial}{\partial t} \theta-D_{T} \Delta \theta=\gamma \frac{W_{F}}{\rho Y_{F 0}}
\end{gathered}
$$

Here $\theta=T / T_{\infty}$, with $T_{\infty}$ the ambient air temperature. The main parameters are: $S=s Y_{F 0} / Y_{0 \infty}$, the air/fuel mass stoichiometric ratio, and $\gamma=q Y_{F 0} / c_{p} T_{\infty}$, the chemical heat release parameter, together with the Lewis numbers $L_{F}$ and $L_{0}$. The reaction term $W_{F} / \rho Y_{F 0}$ will be written in the form

$$
W_{F} / \rho Y_{F 0}=y_{F} y_{0} t_{0}^{-1} e^{\frac{E}{F T_{\infty}} \frac{\theta-1}{\theta}}
$$

involving the non-dimensional activation energy $E / R T_{\infty}$ and the chemical time $t_{0}=\left(Y_{0 \infty} B\right)^{-1} e^{E / R T_{\infty}}$, evaluated at the initial air temperature $T_{\infty}$. 
The system of equations (3)-(5) will be solved, for $x \in R^{3}$ and $t>0$, with the initial conditions

$$
t=0: y_{F}=y_{F I}(x / L), y_{0}=y_{0 I}(x / L), \theta=\theta_{I}(x / L)
$$

where $y_{F I} \leq 1, y_{0 I} \leq 1$ and $\theta_{I}$ are non-negative continuous functions of the space variable $x$, involving a scale $L$, characterizing the size of the fuel pocket; we shall consider $y_{F I}, 1-y_{o I}$ and $\theta_{I}-1$ to be of bounded support. See Fig.1 for a sketch of the distributions.

The existence and uniqueness of the solution of the problem (3)-(7) for $t>0$ is well known; with $y_{F}, y_{0}$ and $\theta$ functions of the class $C_{\infty}$.

The system will be written below in non-dimensional form, measuring $x$ with $L$, and $t$ with the diffusion time $t_{d}=L^{2} / D_{T}$.

From Eq.(3) and (4) we shall first derive the conservation equation

$$
\frac{\partial}{\partial t}\left(S y_{F}-y_{0}\right)-\Delta\left(S y_{F} / L_{F}-y_{0} / L_{0}\right)=0
$$

free from the reaction term. A similar conservation equation, namely,

$$
\frac{\partial}{\partial t}\left(\gamma y_{F}+\theta\right)-\Delta\left(\gamma y_{F} / L_{F}+\theta\right)=0
$$

can be derived from Eqs (3) and (5).

These equations must be solved, for $t>0$, together with the non-dimensional form of Eq (3)

$$
\frac{\partial}{\partial t} y_{F}-\frac{1}{L_{F}} \Delta y_{F}=-\delta y_{F} y_{0} e^{\left(E / R T_{\infty}\right)(\theta-1) / \theta}
$$

and the non-dimensional form (with $L=1$ ) of the initial conditions (7). The non-dimensional Damköhler number $\delta=t_{d} / t_{0}$ is the ratio of the diffusion and chemical times.

Notice that when the mass diffusivities are equal to the thermal diffusivity $\left(L_{F}=L_{0}=1\right)$, Eqs (8) and (9) reduce to the same conservation equation

$$
\frac{\partial W}{\partial t}-\Delta W=0
$$


for the "conserved" scalars $\left(S y_{F}-y_{o}\right)$ and $\left(\gamma y_{F}+\theta\right)$.

We shall present, in the following, the asymptotic form of the solution of the problem (7)-(10) for large values of the Damköhler number, $\delta \gg 1$.

\section{Fast reaction stage for $\delta \gg 1$}

The solution of the problem (7)-(10) for $\delta \gg 1$ involves a first reactive stage for times of order $t_{c}$; or, when measured with $t_{d}$, at non-dimensional times $t$ of order $1 / \delta$ such that $\tau$, defined by

$$
\tau=t \delta
$$

is of order unity.

When the system (8)-(10) is written in terms of the time variable $\tau$, appropriate to the first stage, the diffusion terms appear multiplied by the factor $1 / \delta$. These terms drop out of the equations when we take the formal limit $\delta \rightarrow \infty$, with the space and time derivatives assumed to be of order unity. In this limit the equations (8)-(10) simplify to

$$
\begin{gathered}
\frac{\partial}{\partial \tau}\left(S y_{F}-y_{0}\right)=0 \\
\frac{\partial}{\partial \tau}\left(\gamma y_{F}+\theta\right)=0 \\
\frac{\partial}{\partial \tau}\left(y_{F}\right)=-y_{F} y_{0} \exp \left\{\left(E / R T_{0}\right)(\theta-1) / \theta\right\}
\end{gathered}
$$

for $y_{F}(x, \tau)$ and the conserved scalars

$$
Y=S y_{F}-y_{0} \quad \text { and } \quad H=\gamma y_{F}+\theta
$$

which, according to Eqs. (12) and (13), do not vary with $\tau$, and are given by the initial conditions

$$
\begin{gathered}
Y=S y_{F}-y_{0}=Y_{I}(x)=S y_{F I}-y_{0 I} \\
H=\gamma y_{F}+\theta=H_{I}(x)=\gamma y_{F I}+\theta
\end{gathered}
$$


These equations can be used with $\mathrm{Eq}$ (14) to calculate the evolution of $y_{F}$ with $\tau$ by means of a quadrature.

For values of $\tau$ moderately large compared with unity, but not of order $\delta$, so that the system of equations (12)-(14) remains a valid representation of the system (8)-(10), $y_{F}, y_{0}$ and $\theta$ will reach the intermediate asymptotic form $y_{F i}, y_{0 i}$ and $\theta_{i}$ defined by Eqs (16) and the chemical equilibrium condition

$$
y_{F} y_{0}=0
$$

corresponding to the asymptotic form of (14) for large $\tau$. In the fuel domain, $\Omega_{F I}$, where $Y_{I}>0$, we will run out of oxygen for $\tau \gg 1$; while in the outer complementary domain $\Omega_{0 I}$, where $Y<0$, we run out of fuel. In the level surface $Y_{I}=0$, where the reactants were initially in stoichiometric proportions, the oxygen and the fuel are depleted simultaneously.

At the end of the first stage, at non-dimensional times $t$ such that $1 / \delta \ll t \ll 1$, $y_{F}=y_{F i}, y_{0}=y_{0 i}, \theta=\theta_{i}$ given by

$$
\begin{array}{ll}
y_{0 i}=0, \quad S y_{F i}=Y_{I}(x) & \text { where } Y_{I} \geq 0 \text { at } \Omega_{F I} \\
y_{F i}=0, \quad y_{0 i}=-Y_{I}(x) & \text { where } Y_{I} \leq 0 \text { at } \Omega_{0 I}
\end{array}
$$

The temperature $\theta_{i}$ at the end of the first stage is given by the relation

$$
\theta_{i}-\theta_{I}=\gamma\left(y_{F I}-y_{F i}\right)
$$

Notice that the functions $y_{F i}, y_{0 i}$ and $\theta_{i}$, appearing in the asymptotic form (18) of the solution of Eqs (12)-(14) for $\tau \rightarrow \infty$, although of the class $C_{0}$, do not belong to $C_{1}$, even if the initial functions $y_{F I}, y_{0 I}$ and $\theta_{I}$ are of the class $C_{\infty}$.

The functions $y_{F i}, y_{0 i}$ and $\theta_{i}$ have discontinuous derivatives normal to the surface $Y_{I}(x)=0$, where $y_{F i}$ and $y_{0 i}$ are simultaneously equal to zero. 


\section{Diffusion-controlled stage for $\delta \gg 1$}

The fuel remaining, with the mass fraction $y_{F i}>0$, at $\Omega_{F I}$ diffuses out of the fuel pocket to meet the oxygen, which diffuses from $\Omega_{0 I}$ to $\Omega_{F I}$. They meet at a surface $\Gamma_{f}$, initially at $Y_{I}(x)=0$, where the reaction takes place in a diffusion controlled way, with negligible concentration (in fact of order $\delta^{-1 / 3}$ ) of the reactants.

If we follow the method proposed by Burke and Schumann (1928) in their analysis of the case $L_{F}=L_{0}=1$, we find that the conservation equations (8) and (9), together with (17), describe the evolution of the concentrations and temperature, for large values of the Damköhler number, during the long diffusive stage when $1 / \delta \ll t \sim 1$. Eq.(17) is obtained from $\mathrm{Eq}(10)$ when taking the formal limit $\delta \rightarrow \infty$; in this limit the functions and the derivatives appearing in the equation are assumed to be of order unity.

The system of equations (8), (9) and (17) are to be solved with the initial conditions

$$
y_{F}=y_{F i}, \quad y_{0}=y_{0 i}, \quad \theta=\theta_{i} \quad \text { at } t=0
$$

as given by Eqs (18).

The solution of this limiting problem, for $\delta \rightarrow \infty$, will no longer involve functions $y_{F}, y_{0}$ and $\theta$ belonging to $C_{\infty}$, but only to $C_{0}$. In the limit $\delta \rightarrow \infty$ of infinite reaction rates the chemical production term in Eq (10) becomes localized, as a Dirac delta function of variable strength, on the flame surface, $\Gamma_{f}$, to be determined in terms of $t$.

The Dirac delta function leads to jumps in the derivatives of $y_{F}, y_{0}$ and $\theta$ normal to the flame surface. However, these jumps are such that the functions

$$
\tilde{Y}=S y_{F} / L_{F}-y_{0} / L_{0} \quad \text { and } \quad \tilde{H}=\gamma y_{F} / L_{F}+\theta
$$

must belong, except at $t=0$, to the class of functions $C_{1}$, with continuous derivatives. This is because there are no Dirac delta source terms in the conservation equations (8) and (9) and, therefore, there are no jumps in the spatial derivatives of $\hat{Y}$ and $\hat{H}$. See the sketch of the distributions in Fig.2. 
For the description of the solution of the problem (8), (9), (17) and (19), during the second stage $t \sim 1$, we shall use as dependent variables $Y$ and $H$, defined by (15), and $\hat{Y}$ and $\hat{H}$ defined by Eq (20). In terms of these variables Eqs(8) and (9) take the form

$$
\begin{aligned}
& \frac{\partial}{\partial t}(Y)-\Delta(\tilde{Y})=0 \\
& \frac{\partial}{\partial t}(H)-\Delta(\tilde{H})=0
\end{aligned}
$$

while the condition (17) implies that

$$
\begin{array}{lll}
y_{0}=0, \quad \dot{S y_{F}}=Y=L_{F} \tilde{Y} & \text { where } \tilde{Y} \geq 0 \\
y_{F}=0, \quad-y_{0}=Y=L_{0} \tilde{Y} & \text { where } \tilde{Y} \leq 0
\end{array}
$$

and, similarly,

$$
\begin{gathered}
\theta=\tilde{H}-\gamma \tilde{Y} / S, \quad H=\tilde{H}+\left(L_{F}-1\right) \gamma \tilde{Y} / S \quad \text { where } \quad \tilde{Y} \geq 0 \\
\theta=H=\tilde{H} \quad \text { where } \quad \tilde{Y} \leq 0
\end{gathered}
$$

Thus we obtain the system of conservation equations (21)-(22), where the pseudo enthalpies $Y$ and $H$ are known continuous, piece-wise linear, functions of $\tilde{Y}$ and $\tilde{H}$ given by the chemical equilibrium relations (23)-(24) derived from (17). The system (21)-(24) must be solved with the initial conditions

$$
t=0: \quad Y=Y_{I}(x)=S y_{F I}-y_{0 I}, H=H_{I}(x)=\gamma y_{F I}+\theta_{I}
$$

The solution of this system will provide us with the temperature and concentration field and, in addition, with the time evolution of the flame surface $\Gamma_{f}$, given by the level surface

$$
\tilde{Y}(x, t)=0
$$

The flame temperature there will be $\theta_{f}=\tilde{H}_{f}$.

Notice that while $\tilde{Y}$ and $\tilde{H}$ belong to $C_{1}$, we can only insure that $Y$ and $H$ belong to $C_{0}$; their spatial derivatives may have jumps at $\Gamma_{f}$. Notice also that if the Lewis numbers are equal to unity the problem simplifies considerably, because 
$Y=\tilde{Y}$ and $H=\tilde{H}$; which, for $t>0$, become again functions of the class $C_{\infty}$, even if they were not so initially.

\section{Concluding remarks}

In many practical combustion systems the reactants are not initially partially mixed, as in the example considered here, but are fed in the form of independet jets into the reacting chamber. The conservation equations must then include convective terms associated with the flow.

After an ignition transient stage, whose memory will disappear during the stationary operation of the chamber, the reaction will be diffusion controlled; and described by a straight-forward generalization of the procedure outlined above, if the characteristic chemical time, at the flame temperature $T_{f}$, is short compared with the diffusion time. An analysis of the internal structure of the flame, where the reaction term is balanced by the diffusion of heat and mass normal to the thin flame, is required to insure, as shown by Liñán (1974), that indeed the reaction is diffusion controlled.

Some direct numerical simulations of turbulent flow fields with diffusion controlled reactions have already been carried out, see Liñán et al. (1994), using the above formulation.

Let us end this note by noticing that, in the combustion literature,the coupling function $Y=S y_{F}-y_{0}$ is replaced by the mixture fraction variable

$$
Z=\left(S y_{F}-y_{0}+1\right) /(S+1)
$$

equal to zero on the air feed stream, and equal to 1 in the fuel feed stream.

The generalized mixture fraction to be used, with $Z$, when the Lewis numbers are not equal to 1 , can be defined as

$$
\tilde{Z}=\frac{S y_{F} / L_{F}-y_{0} / L_{0}+1 / L_{0}}{S / L_{F}+1 / L_{0}}=\frac{\tilde{S} y_{F}-y_{0}+1}{\tilde{S}+1}
$$

where $\tilde{S}=S L_{0} / L_{F}$. 
The conservation equation (21), when written in terms of $\tilde{Z}$, takes the form

$$
\frac{\partial Z}{\partial t}-\frac{1}{L_{m}} \Delta \tilde{Z}=0
$$

where $L_{m}=L_{0}(1+S) /(1+\tilde{S})$ is a mean value of the Lewis number, and $Z$ is a continuous, piece-wise linear, function of $\tilde{Z}$. The flame surface lies, where $y_{F}$ and $y_{0}$ are simultaneously equal to zero, at

$$
\tilde{Z}=\tilde{Z}_{s}=1 /(\tilde{S}+1)
$$

where $Z=Z_{s}=1 /(S+1)$. The chemical equilibrium condition, $y_{F} y_{0}=0$, leads to

$$
\begin{gathered}
y_{F}=0, \quad \text { where } \quad 1-y_{0}=\tilde{Z} / \tilde{Z}_{s}=Z / Z_{s} \geq 0 \\
y_{0}=0, \quad \text { where } \quad y_{F}=\left(\tilde{Z}-\tilde{Z}_{s}\right) /\left(1-\tilde{Z}_{s}\right)=\left(Z-Z_{s}\right) /\left(1-Z_{s}\right) \geq 0
\end{gathered}
$$

Thus providing a relation between $Z$ and $\tilde{Z}$.

Acknowledgment. This research was carried out under partial support of the CICYT, under Contract No. PB94-0400, and also by the INTA, under Contract 4070-0036/1995.

\section{References}

1. Burke, S.P. and Schumann, T.E.W. (1928) Diffusion flames. Ind. Eng. Chem.20, 998-1004.

2. Liñán, A. (1974) The asymptotic structure of counter-flow diffusion flames for large activation energies. Astronautica Acta 1, 1007-1031.

3. Liñán, A. (1991a) The structure of diffusion flames. In Fluid Dynamical Aspects of Combustion Theory. Ed. by M. Onofri and A. Tesev. Longman Scientific and Technical pp11-29

4. Liñán, A. (1991b) "El Papel de la Mecánica de Fluidos en los Procesos de Combusti $\not n "$. Real Academia de Ciencias, Madrid, Spain.

5. Liñán, A. and Williams, F.A. (1993) "Fundamental Aspects of Combustion". Oxford Univ. Press.

6. Liñán, A.; Orlandi, P.; Verzicco, R. and Higuera, F.J. (1994) Effects of non-unity Lewis numbers in diffusion flames. Proc. of the Summer Program 1994. Center of Turbulence Research. Stanford University.

7. Williams, F.A. (1985) "Combustion Theory". Benjamin Cumm ings. 


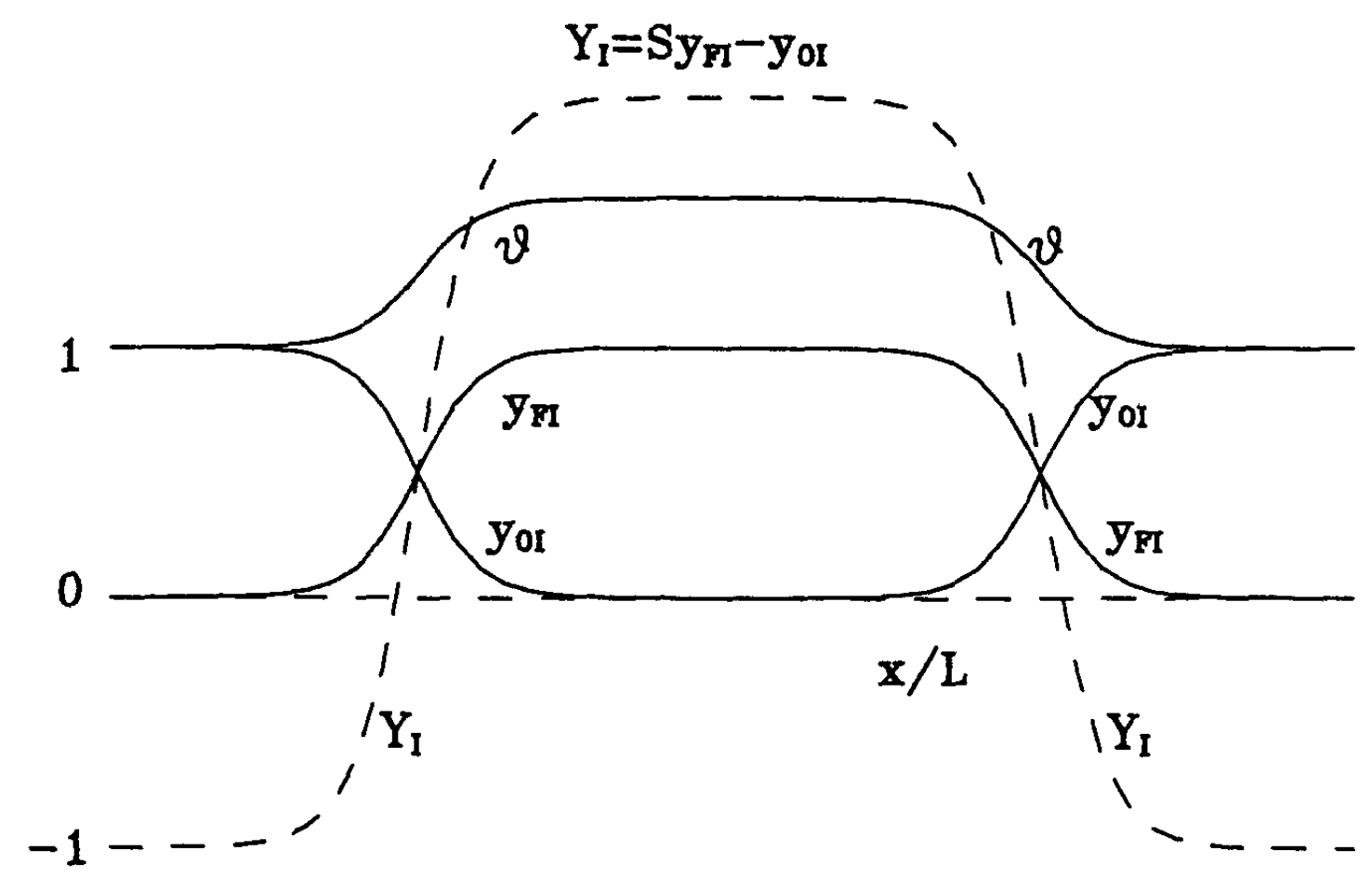

Figure 1. Initial distributions of the mass fractions and temperature for a fuel pocket of size $L$.

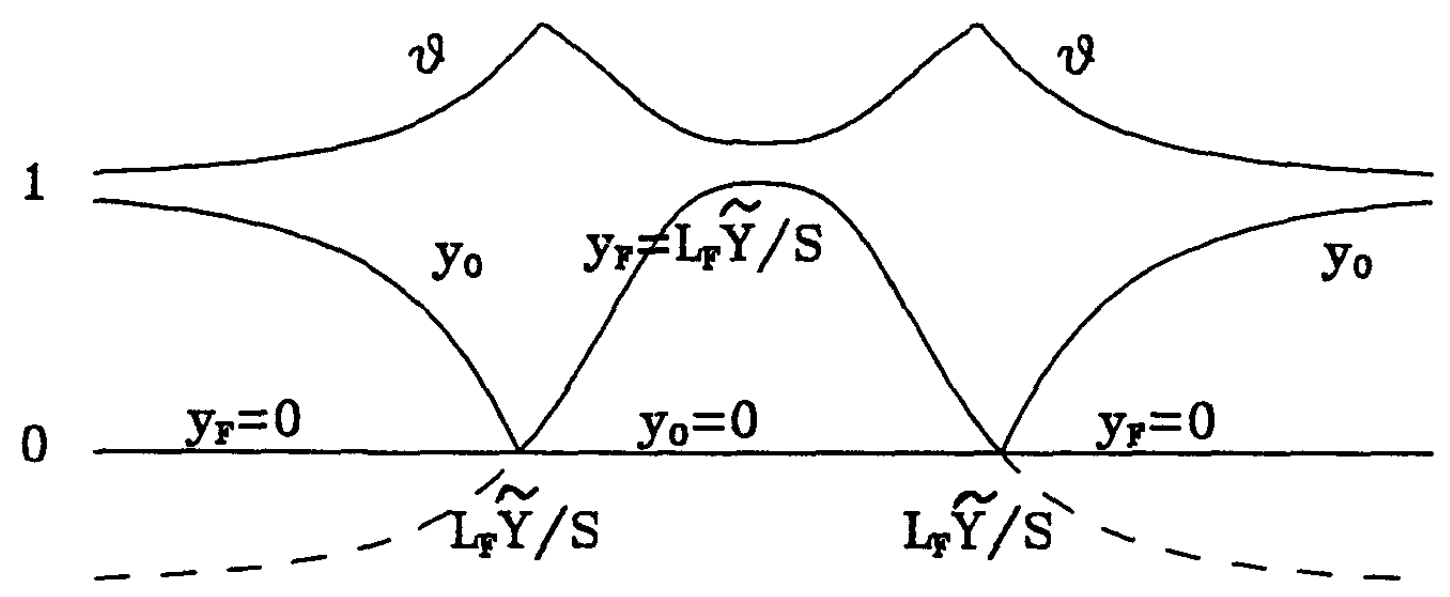

Figure 2. Temperature and mass fraction distribution at a later time during the diffusion controlled stage. 